\title{
Antioxidant activity of 5-aminosalicylic acid against lipid peroxidation in the presence of vitamins $\mathrm{C}$ and $\mathrm{E}$
}

\author{
Elisabete Gonçalves a, Leonor M. Almeida ${ }^{\text {a,b }}$, Teresa C.P. Dinis ${ }^{\text {a,b,* }}$ \\ ${ }^{a}$ Laboratório de Bioquímica, Faculdade de Farmácia, Universidade de Coimbra, R. do Norte, 3000 Coimbra, Portugal \\ ${ }^{\mathrm{b}}$ Centro de Neurociencias, Universidade de Coimbra, 3000 Coimbra, Portugal
}

Received 15 April 1998; received in revised form 25 June 1998; accepted 26 June 1998

\begin{abstract}
It is now recognized that the antioxidant defences in the colonic mucosa from patients with inflammatory bowel disease (IBD) are particularly low. On account of this we studied the antioxidant capacity of 5-ASA, the first choice therapy in this pathological situation, in combination with the endogenous antioxidants vitamins C (ASC) and E $(\alpha$-tocopherol $(\alpha-\mathrm{T}))$ against lipid peroxidation in phosphatidylcholine (PC) liposomes as a model membrane. The oxidative process was initiated by peroxyl radicals generated at different sites in liposomes by thermal decomposition of azocompounds. 5-ASA interacts additively with ASC in the protection of membranes against peroxyl radicals generated in the aqueous phase, as evaluated by the oxygen consumption or formation of conjugated dienes. HPLC analysis of 5-ASA indicates that this drug is consumed at a constant rate throughout the oxidation reaction, but in the presence of ASC there is a lag phase of its consumption, denoting that ASC affords an efficient protection to 5-ASA. On the other hand, 5-ASA and ASC cooperate, in an additive way, in the protection of $\alpha$-T. When the oxidation starts within the membrane, $\alpha-\mathrm{T}$ is the preferential target of peroxyl radicals, but 5-ASA and ASC also interact additively in sparing of $\alpha-\mathrm{T}$, increasing not only the inhibition period of oxidation reaction, but also decreasing significantly the rate of $\alpha$-T consumption. (C) 1998 Elsevier Science B.V. All rights reserved.
\end{abstract}

Keywords: 5-Aminosalicylic acid; Vitamin C; Vitamin E; Inflammatory bowel disease; Antioxidant activity

\section{Introduction}

5-Aminosalicylic acid (5-ASA) is the drug used

\footnotetext{
* Corresponding author. Fax: + 35139 827940; e-mail Tcpdinis@gemini.ci.uc.pt
}

as a first choice therapy in the treatment of inflammatory bowel diseases (IBD) (Rhodes et al., 1997). Among the multiple mechanisms of action of 5-ASA, its antioxidant activity is obviously important, considering that reactive oxygen species (ROS) have been implicated in many inflammatory disorders, including those of the

0378-5173/98/\$ - see front matter (C) 1998 Elsevier Science B.V. All rights reserved.

PII S0378-5173(98)00211-7 
gastrointestinal tract (Conner et al., 1996; Nielsen and Rask-Madsen, 1996).

Actually, it has been accepted that ROS are produced in excess by the inflamed mucosa, whose predominant sources are probably activated mucosal phagocytic leukocytes (Grisham and Granger, 1988). Excess production of ROS may exceed cellular cytoprotective mechanisms and may be highly toxic to cells, being related with the tissue injury developed in the intestinal mucosa of IBD patients (Keshavarzian et al., 1992).

On the other hand, as in the colonic mucosa there is relatively low tissue levels of endogenous antioxidants, it has been suggested that the efficacy of the treatment could be related to the drug antioxidant action (Lih-Brody et al., 1996; Millar et al., 1996). Indeed, the ability of 5-ASA in scavenging different reactive species produced during inflammation, such as superoxide radicals (Allgayer et al., 1994), hydroxyl radicals (Ahnfelt-Ronne et al., 1990), hydrogen peroxide (Miles and Grisham, 1994), hypochlorous acid (Aruoma et al., 1987) and peroxyl radicals (Dinis et al., 1994), has already been reported.

Beyond its direct antioxidant activity against such reactive species, 5-ASA could also play an important role by interacting with endogenous antioxidants. Previously, we have demonstrated a potential synergistic interaction between 5-ASA and vitamin $\mathrm{E}(\alpha$-tocopherol $(\alpha-\mathrm{T}))$, the major lipid-soluble antioxidant in protecting lipids from peroxidative damage (Gonçalves et al., 1998). Since recent investigations have shown that the proportion of reduced ascorbate present in the mucosa from IBD patients is very low (Buffinton and Doe, 1995a), in this work we explored the possible interactions between 5-ASA and vitamin C (ascorbic acid) against lipid oxidation. 5-ASA and ascorbic acid (ASC) are hydrophilic compounds with the same location relative to the membrane, so a putative cooperation between them could be another important contribution to the action mechanism of 5-ASA in IBD. Moreover, 5-ASA and ASC, each one per se, protect $\alpha$-T from radical attack, so when combined such an effect may be strongly enhanced.
Thus, the aim of this work was to study the potential cooperative interaction between 5-ASA and vitamin C (ASC) against lipid oxidation in soybean phosphatidylcholine liposomes, used as a membrane model system, with or without $\alpha-T$, induced by peroxyl radicals generated either in the aqueous phase or in the lipid phase, by thermal decomposition of 2,2'-azobis-(2-amidinopropane hydrochloride) (AAPH) or 2,2'-azobis-(2,4dimethylvaleronitrile) (AMVN). The azocompounds AAPH and AMVN are excellent free radical initiators for the quantitative study of lipid peroxidation and its inhibition, because they are able to generate peroxyl radicals at a known and constant rate and at a specific site (Niki, 1990). Therefore, depending on the azoinitiator used, it is possible to clarify the inhibitory behaviour of the antioxidant tested against lipid peroxidation. The extent of membrane lipid oxidation was followed by measuring the oxygen consumption and conjugated diene hydroperoxides production and, additionally, to complement the study, HPLC determinations of 5-ASA and $\alpha$-T were performed throughout the oxidation reaction.

\section{Materials and methods}

\subsection{Chemicals}

Commercial soybean phosphatidylcholine (PC), 5-ASA and $\alpha$-T were obtained from Sigma (St. Louis, MO). Ascorbic acid (ASC) was obtained from Fluka (Switzerland). The azocompounds 2,2'-azobis(2-amidinopropane hydrochloride) (AAPH) and 2,2'-azobis(2,4-dimethylvaleronitrile) (AMVN) were purchased from Polysciences (Warrington, PA). AAPH was used as supplied and AMVN was used after purification by recrystallisation in cold methanol. All the other reagents and chemicals were of analytical grade for biochemical purposes or HPLC grade. In order to prevent metal contamination, all the solutions were prepared in ultrapure MilliQ water. 5-ASA and ASC solutions were prepared freshly every day, protected from light and maintained in ice during the experiments. 


\subsection{Liposome preparation}

Soybean PC unilamellar or multilamellar liposomes were used as suitable membrane models for lipid peroxidation studies.

\subsubsection{Multilamellar vesicles ( $M L V S$ )}

Multilamellar liposome suspensions were prepared by adding aliquots of chloroformic solution of soybean PC and, when required, aliquots of stock solutions of the lipophilic additives AMVN and $\alpha-T$, in a pear-shaped flask. Solvents were evaporated slowly, in a rotary evaporator, to obtain a thin film on the flask wall. An appropriate amount of phosphate-buffered saline solution (20 $\mathrm{mM} \mathrm{Na} \mathrm{HPO}_{4}, 100 \mathrm{mM} \mathrm{NaCl}, \mathrm{pH}$ 7.4) (PBS) containing $100 \mu \mathrm{M}$ ethylenediaminetetraacetic acid (EDTA) was added into the flask and the film was peeled off by vortexing to obtain a milky aqueous suspension of multilamellar liposomes (MLVs) (Yamamoto et al., 1984).

\subsubsection{Large unilamellar vesicles ( LUVS)}

Unilamellar liposomes (LUVs) were prepared by extrusion of the MLV suspension, using the Avestin Liposofast (Avestin, Ottawa, Canada) small-volume extrusion device, provided with a polycarbonate membrane $200 \mathrm{~nm}$ pore size. After seven extrusion steps, back and forth, by simply applying a manual pressure, the resulting LUV suspension proved to be a homogeneous population of large unilamellar liposomes, as measured by photon correlation spectroscopy (MacDonald et al., 1991; Fiorentini et al., 1994)

\subsection{Lipid peroxidation procedures}

Lipid peroxidation of soybean PC liposomes was induced initially either outside or within the bilayer by peroxyl radicals, generated at a constant rate, by thermal degradation of azocompounds (Niki, 1990), in the presence or absence of antioxidants and followed by measuring the oxygen consumption and conjugated diene hydroperoxides production (Slater, 1984).

Unilamellar liposomes (LUVs) were used when lipid peroxidation was stimulated in the aqueous phase, outside the vesicle, by AAPH at $37^{\circ} \mathrm{C}$ in air. When AMVN was the initiator, we could not incorporate it into the unilamellar structure in the required concentration, so multilamellar liposomes (MLVs) were used as membrane models, and the oxidation reactions were carried out at $56^{\circ} \mathrm{C}$ in air. The water-soluble compounds, 5-ASA and ASC, were added, when necessary, in a minimum volume of a PBS solution containing 100 $\mu \mathrm{M}$ EDTA, immediately before starting the oxidation reaction.

\subsubsection{Oxygen consumption}

The rate of oxygen consumption was measured continuously with a Clark-type oxygen electrode (YSI Model 5331, Yellow Springs Inst.) provided with an automatic recording apparatus. The reaction mixture was introduced in a closed glass vessel protected from light, thermostatted and maintained under agitation with a magnetic stirrer. When AAPH was used as initiator, reactions were started by addition of AAPH $(10 \mathrm{mM}$ final concentration), in a small volume of PBS, to $1 \mathrm{ml}$ of LUV suspension (800 $\mu \mathrm{M}$ PC) (Dinis et al., 1994). 5-ASA and ASC were added 1 min before the addition of AAPH, and the vessel was always kept at $37^{\circ} \mathrm{C}$. When AMVN $(500 \mu \mathrm{M}$ final concentration) was used as initiator, the reactions were started when the MLV suspension $(1.5 \mathrm{mM}$ PC) attained the reaction temperature inside the vessel thermostatted at $56^{\circ} \mathrm{C}$. The hydrophilic antioxidants were added immediately before this starting point.

\subsubsection{Conjugated diene hydroperoxide production}

Soybean PC liposomes (5 mM final concentration) were incubated in a water bath under air, at 37 or $56^{\circ} \mathrm{C}$, depending on the azocompound used, AAPH $(20 \mathrm{mM})$ or AMVN $(1.67 \mathrm{mM})$, respectively. Aliquots of liposomes $(20 \mu 1)$ were taken at 10-min intervals and dissolved in $2 \mathrm{ml}$ of absolute ethanol directly in $1 \mathrm{~cm}$ (light path) quartz cuvettes (Tesoriere et al., 1996). The reference cuvette lacked the liposome suspension but contained an identical volume of PBS buffer. At the oxidation time intervals, spectra were then recorded in the range $210-300 \mathrm{~nm}$, using a Perkin-Elmer Lambda 6. Conjugated diene hydroperoxide production was evaluated by the 
height of the minimum peak at $233 \mathrm{~nm}$ (in arbitrary units) in the second derivative difference spectra, which allowed the resolution of the shoulder at $233 \mathrm{~nm}$ in the absorbance spectra (Corongiu et al., 1989). The difference spectra were obtained after subtracting the spectra recorded at 'time 0' from those recorded at different times. The addition of 5-ASA or ASC to the liposomal system was performed in a similar way to that described above for oxygen consumption experiments.

The inhibition periods in the presence of 5ASA, ASC or $\alpha-T$, individually or combined, were determined graphically from the profiles of oxygen consumption or of conjugated dienes formation by the coordinates of the interception of the tangents to the inhibited and uninhibited rates of oxidation.

\subsection{HPLC analysis of 5-ASA and $\alpha-T$}

The consumption of either 5-ASA or $\alpha$-T during lipid peroxidation was followed by HPLC (Beckman System Gold, UV detector model 166), using a Lichrospher 100 RP-18 (particle size, 5 $\mu \mathrm{m})$ column $(125 \times 4 \mathrm{~mm}$ Merck). For $\alpha-\mathrm{T}$ analysis the mobile phase consisted of methanol $(100 \%)$, at a flow rate of $2 \mathrm{ml} / \mathrm{min}$, and UV detection at $290 \mathrm{~nm}$ (Lang et al., 1986), while for 5-ASA the eluent was $0.1 \mathrm{M} \mathrm{KH}_{2} \mathrm{PO}_{4}(\mathrm{pH} \mathrm{4.4)/}$ methanol (95:5 by volume), at a flow rate of 1 $\mathrm{ml} / \mathrm{min}$ (Fischer and Klotz, 1994), and UV detection at $240 \mathrm{~nm}$. These determinations were performed in parallel with the oxygen consumption and, therefore, the experimental conditions of oxidation reaction were those stated for this assay. $\alpha$-T was previously extracted from the membranes essentially as reported by Burton et al. (1985). Briefly, a 2-ml aliquot of the liposome suspension was removed, at different oxidation times, from the reaction mixture and immediately extracted into hexane. After extraction, an exact volume of the organic phase was removed, evaporated and the lipidic extract was dissolved in a minimum volume of methanol $(200 \mu 1)$. For 5-ASA analysis, 2-ml aliquots of liposome suspension were also taken from the reaction mixture, at different times along the oxidation reaction, and submitted to ultracentrifugation in a Beckman TL-100 ultracentrifuge (Beckman Instruments, Palo Alto, CA), at $90000 \mathrm{rpm}$, for $1 \mathrm{~h}$ at $4^{\circ} \mathrm{C}$. Under these conditions, no lipid was detected in the supernatant, as revealed by spectrophotometric analysis (data not shown). The samples were stored at $-80^{\circ} \mathrm{C}$ until HPLC analysis.

\section{Results}

\subsection{AAPH-induced oxidation of soybean PC liposomes}

\subsubsection{Inhibitory effects of 5-ASA in combination with vitamin $C$}

The antioxidant activity of 5-ASA, in combination with ASC, against the oxidation of soybean PC liposomes initiated in the aqueous phase, by thermal degradation of AAPH, is shown in Fig. 1. The results indicate the effects obtained in the absence (control) and presence of 5-ASA and ASC, either alone or combined, on the rates of oxygen uptake (A) and on the conjugated diene hydroperoxides formation (B). The generation of peroxyl radicals from AAPH induces a significant oxidation of phosphatidylcholine, as indicated by the appreciable rates of oxygen uptake (A) and of production of conjugated dienes (B), since they are able to subtract hydrogen atoms from polyunsaturated acyl chains, yelding lipid radicals that lead to the propagation reactions. Either 5-ASA or ASC scavenge efficiently the peroxyl radicals initially generated in the aqueous phase. Actually, clear initial inhibition periods (lag phases) of either oxygen consumption (A) or production of conjugated dienes (B) are detected in the presence of $10 \mu \mathrm{M}$ 5-ASA or ASC, which are followed with a resumption of the propagation rate, i.e. the oxidation proceeds at a similar rate to that without antioxidants. Therefore, a typical chainbreaking antioxidant behaviour is observed for both antioxidants, which is, according to previous data, related to their antioxidant properties (Beyer, 1994; Dinis et al., 1994; Gonçalves et al., 1998). Additionally, as evidenced in Fig. 1, 5-ASA suppresses the lipid oxidation more efficiently than ASC, causing a longer initial lag phase, in 
both assays. Moreover, under similar experimental conditions, the combination of 5-ASA and ASC leads to an additive antioxidant effect. In fact, as documented in Fig. 1, when we tested the two compounds together, the length of the initial inhibition period was close to the sum of the individual inhibition periods produced by each one. Therefore, the two hydrophilic antioxidant
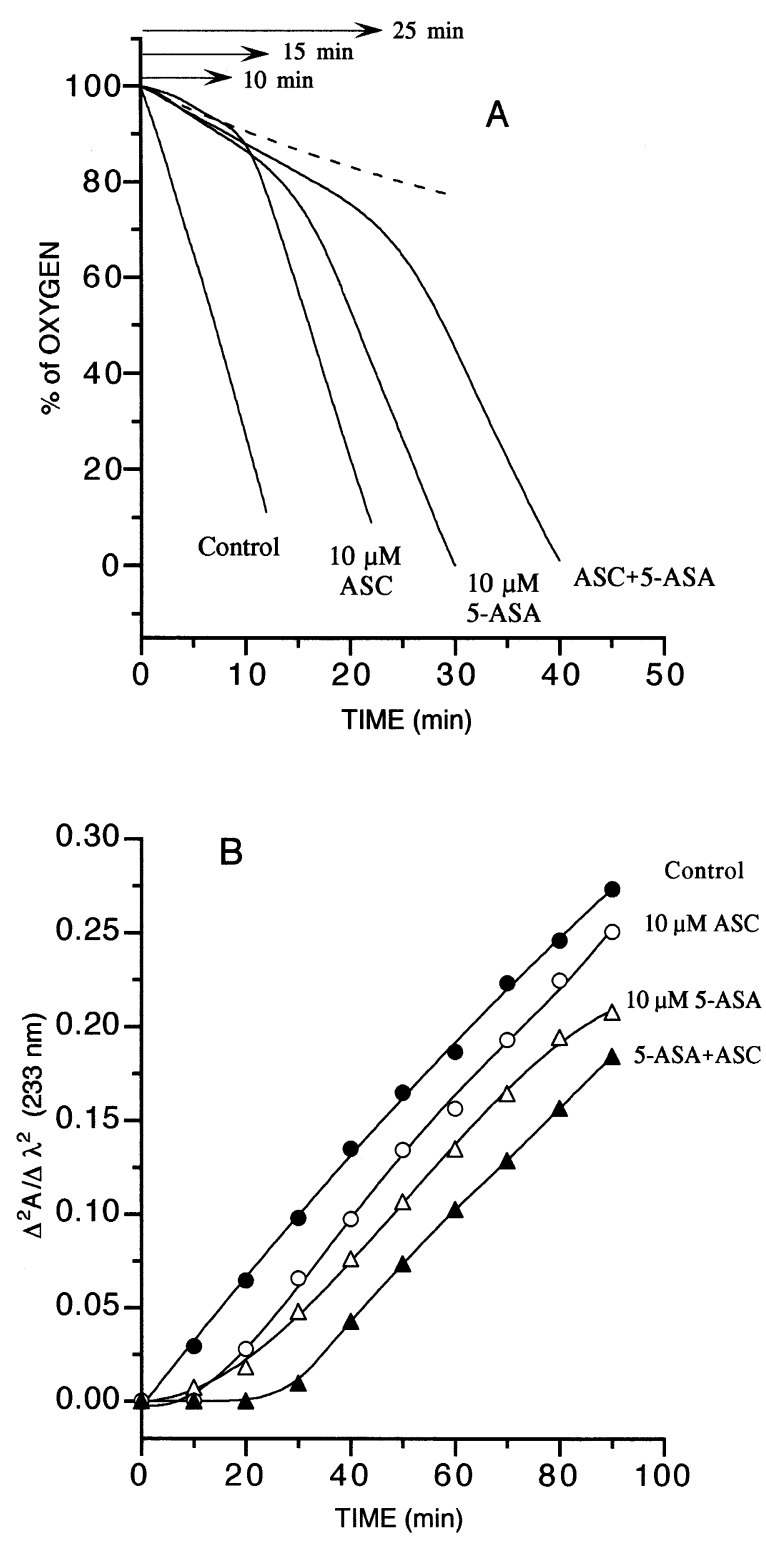

Fig. 1. compounds react with the peroxyl radicals generated in the aqueous phase from AAPH, but their effects are only additive, i.e. both compounds act independently of each other.

\subsubsection{Inhibitory effects of 5-ASA in combination with vitamins $C$ and $E$}

As shown in Fig. 2, the incorporated $\alpha$-T (2.5 $\mu \mathrm{M}$ ) alone (control) induces an initial lag phase of the oxygen consumption revealing the capacity of $\alpha-\mathrm{T}$ to scavenge efficiently the peroxyl radicals generated in the aqueous phase. This initial inhibition period, due to $\alpha-\mathrm{T}$ (13 $\mathrm{min})$, increases when we add to the lipid suspension, just before AAPH, 5-ASA or ASC $(10 \mu \mathrm{M})$, individually or in combination. The increases in the lag phase due to $\alpha$-T, by 5 -ASA (15 $\mathrm{min})$ or ASC (19 min) individually, are according to the results previously reported (Doba et al., 1985; Gonçalves et al., 1998).

When the two hydrophilic compounds are added together, the extent of the inhibition period (45 min) is the sum of the individual effects in this liposomal system, i.e. similar to the results referred to above, only an additive inhibition is observed.

Identical results have been obtained by measuring the lipid oxidation by the production of conjugated dienes (results not shown).

Fig. 1. Inhibition of AAPH-initiated oxidation in soybean PC liposomes by a combination of 5-ASA and ASC, as measured by oxygen consumption (A) and formation of conjugated dienes (B). (A) Rates of oxygen consumption during PC oxidation $(800 \mu \mathrm{M})$ by AAPH $(10 \mathrm{mM})$, added at time zero to lipid suspensions, in the absence (control) and presence of 10 $\mu \mathrm{M} 5$-ASA or $10 \mu \mathrm{M}$ ASC, individually or together $(10 \mu \mathrm{M}$ 5 -ASA $+10 \mu \mathrm{M}$ ASC). The dashed line represents a control experiment in the absence of liposomes. The arrows indicate the inhibition times of oxygen consumption (10 min for ASC, $15 \mathrm{~min}$ for 5-ASA and $25 \mathrm{~min}$ for ASC + 5-ASA). (B) Formation of conjugated dienes as a function of time, as evaluated by second derivative spectrophotometry in the absence (control, ๑) or presence of $10 \mu \mathrm{M}$ ASC $(\bigcirc), 10 \mu \mathrm{M}$ 5-ASA $(\triangle)$ and $10 \mu \mathrm{M}$ 5-ASA $+10 \mu \mathrm{M}$ ASC ( $\mathbf{\Delta})$. Experimental conditions are described in Section 2. The graphics show typical experiments representative of at least three independent experiences. 


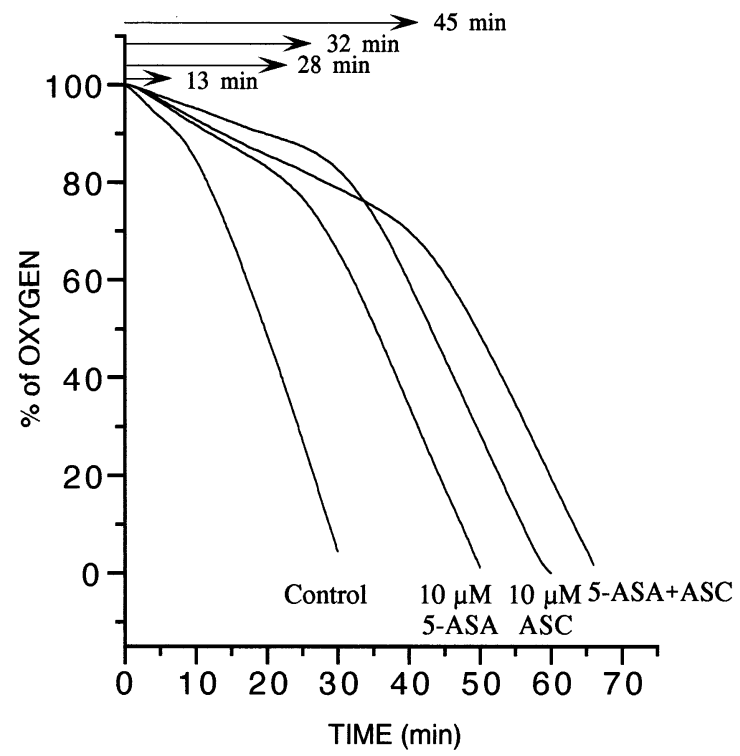

Fig. 2. Effects of 5-ASA and ASC on AAPH-initiated oxidation of PC liposomes containing $\alpha-\mathrm{T}(2.5 \mu \mathrm{M})$ previously incorporated. Rates of oxygen consumption during lipid oxidation in the absence (control) and presence of $10 \mu \mathrm{M}$ 5-ASA, $10 \mu \mathrm{M} \mathrm{ASC}$ and $10 \mu \mathrm{M}$ 5-ASA $+10 \mu \mathrm{M}$ ASC. The arrows indicate the inhibition times of oxygen consumption due to the presence of $\alpha$-T alone (13 min), and combined with 5-ASA (28 $\mathrm{min}$ ), and ASC (32 min), individually or together (45 min). Experimental conditions are described in Section 2. Recordings are representative assays of at least three independent experiments.

\subsubsection{Consumption of 5-ASA and $\alpha-T$}

Antioxidant consumptions were evaluated throughout the oxidation reaction by HPLC anal-

Fig. 3. Time courses of the 5-ASA consumption during AAPH-induced peroxidation in the absence $(\triangle)$ and presence of $10 \mu \mathrm{M}$ ASC ( $\mathbf{\Delta})$ in PC liposomes without (A) or with $\alpha-\mathrm{T}$ (B). (A) The oxidation reaction was carried out in soybean PC liposomes and 5-ASA and ASC were added before starting the oxidation reaction. (B) The reaction was performed in the same experimental conditions, but the liposomes contained $2.5 \mu \mathrm{M}$ $\alpha$-T previously incorporated. Liposomes ( $800 \mu \mathrm{M}$ PC) were exposed to $10 \mathrm{mM} \mathrm{AAPH}$ and, at indicated times, aliquots were withdrawn for 5-ASA extraction and HPLC measurement, as indicated in Section 2. Note that when ASC is absent in the reaction medium, the consumption of 5-ASA is rapid and almost linear. However, in the presence of ASC, an inhibition period (20 $\mathrm{min})$ in the 5-ASA consumption occurs, either in the absence or presence of $\alpha$-T. Each point in the curves represents the mean of triplicate results. Deviations are generally encompassed by the size of the symbols. ysis, as indicated in Section 2. 5-ASA determinations were performed either when 5-ASA was used alone or combined with the two endogenous antioxidants, vitamins $\mathrm{C}$ and E. Fig. 3 shows the time courses of 5-ASA consumption in the absence (open symbols) and presence of ASC (full symbols) during AAPH-induced lipid peroxidation in soybean PC liposomes without (A) or with
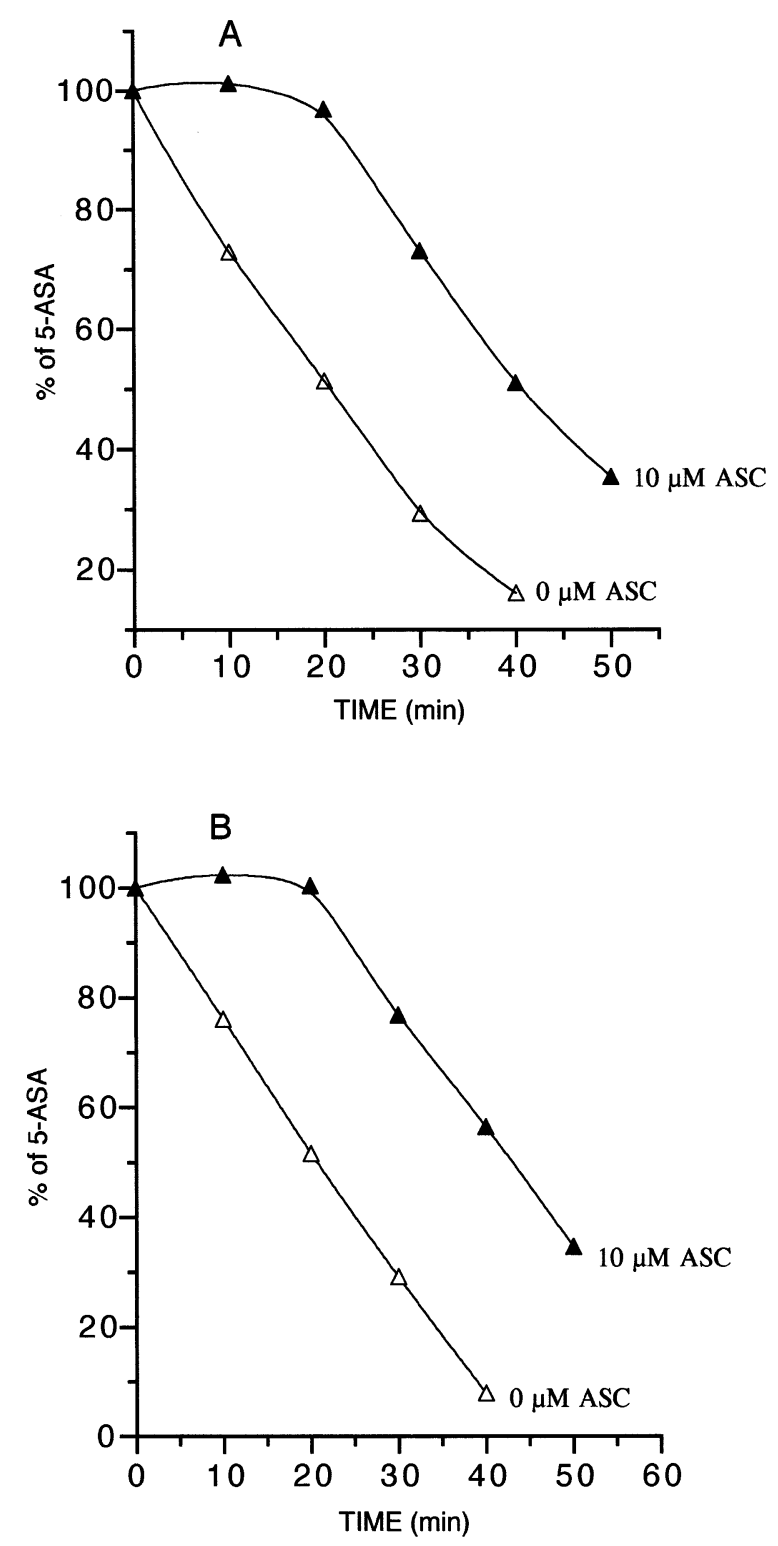

Fig. 3. 
$\alpha-\mathrm{T}$ previously incorporated into the membranes (B). Independently of the presence of $\alpha$-T, 5-ASA is consumed, at a constant rate, throughout the oxidation reaction, evidencing the efficient ability of 5-ASA to trap peroxyl radicals generated from AAPH thermal decomposition. However, when we add ASC to the reaction mixture, a clear lag phase (approximately $20 \mathrm{~min}$ ) of 5-ASA consumption can be detected, either in the presence (Fig. 3B) or absence (Fig. 3A) of $\alpha$-T. These results indicate that ASC reacts with peroxyl radicals faster than 5-ASA, protecting this compound from the attack of radicals when both compounds are simultaneously in the hydrophilic region of the liposomal system.

Moreover, in the assays with liposomes containing $\alpha$-T, we also followed the $\alpha$-T consumption throughout the oxidation reaction by HPLC analysis. As shown in Fig. 4, when 5-ASA and ASC are combined, a relatively long inhibition period of $\alpha$-T consumption is observed. However, the extent of this protective period is only similar to the sum of the individual lag periods due to ASC and 5-ASA separately. Therefore, these results correlate well with those obtained by the oxygen consumption assay (Fig. 2) and prompted us to conclude that 5-ASA and ASC inhibit, in an additive manner, the lipid peroxidation initiated in the aqueous phase, sparing $\alpha$-T incorporated inside the membranes from radical attack.

\subsection{AMVN-induced oxidation of soybean PC liposomes}

\subsubsection{Combination of 5-ASA with vitamins $C$ and $E$}

To clarify the potential cooperative interactions between 5-ASA and the endogenous antioxidants ascorbic acid and $\alpha$-T, we also studied the combined inhibitory effects of the hydrophilic compounds 5-ASA and ASC, against the lipid oxidation initiated by peroxyl radicals generated inside the lipid bilayer in PC liposomes containing $\alpha$-T. Fig. 5 shows the results obtained when we evaluated the lipid oxidation by the measurement of oxygen consumption. As formerly demonstrated, $\alpha$-T suppresses efficiently the initial rate of oxygen consumption, producing a clear inhibi-

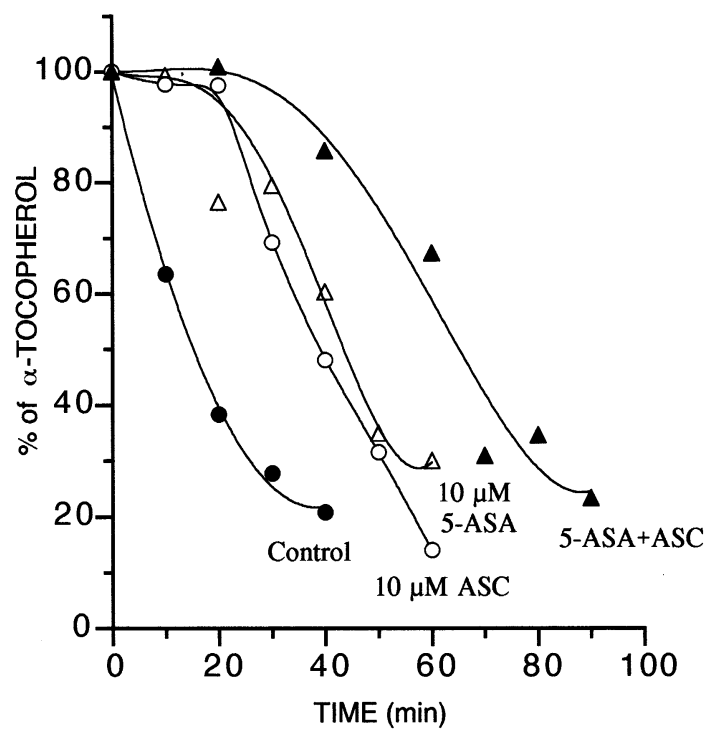

Fig. 4. Time courses of the consumption of $\alpha$-T in soybean PC liposomes (800 $\mu \mathrm{M}$ PC, $2.5 \mu \mathrm{M} \alpha$-T) during AAPH-induced peroxidation in the absence $(\bullet)$ and presence of $10 \mu \mathrm{M}$ ASC $(\bigcirc), 10 \mu \mathrm{M}$ 5-ASA $(\triangle)$ and 5-ASA + ASC $(\boldsymbol{\Delta})$ added just before starting the oxidation reaction with AAPH. Note that, in the presence of the hydrophilic compounds, an inhibition period in the consumption of $\alpha-\mathrm{T}$ occurs. Each point represents the mean of triplicate assays, with an error lesser than $3 \%$.

tion period on the initial rate of oxidation (10 min). Furthermore, when we add each hydrophilic antioxidant, 5-ASA or ASC, separately, to the liposome suspension, an increase in the inhibition period is obtained for each one (7 and $10 \mathrm{~min}$ for 5-ASA and ASC, respectively). On the other hand, a longer prolongation of the inhibition period, due to $\alpha-T$, is detected when 5-ASA and ASC are added, together, to the same liposomal preparation. However, this additional period of time $(16 \mathrm{~min})$ is only similar to the sum of the prolongations due to each hydrophilic antioxidant, independently. Identical results were obtained by measuring formation of conjugated diene hydroperoxides (results not shown).

\subsubsection{Consumption of $\alpha$-tocopherol}

Additionally, we measured the $\alpha$-T consumption during the oxidation reaction, in the absence or presence of 5-ASA and ASC. The results ob- 


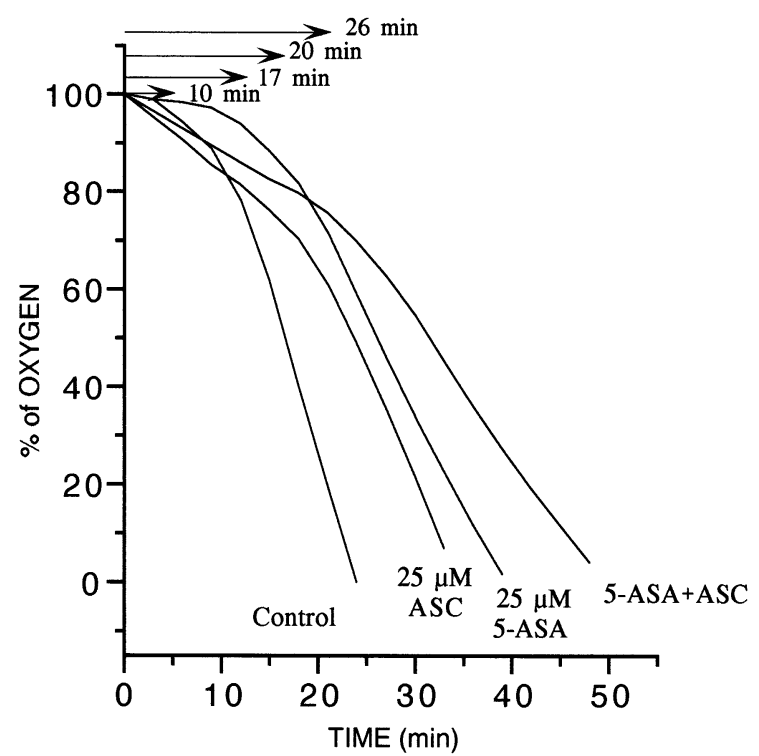

Fig. 5. Effects of 5-ASA and ASC on AMVN-induced oxidation of PC liposomes (1.5 mM PC) containing $\alpha$-T $(2.5 \mu \mathrm{M})$. Rates of oxygen consumption during lipid oxidation in the absence of the hydrophilic compounds (control) and presence of $25 \mu \mathrm{M}$ ASC, $25 \mu \mathrm{M}$ 5-ASA and $25 \mu \mathrm{M}$ 5-ASA $+25 \mu \mathrm{M}$ ASC, as indicated beside the traces. The arrows indicate the inhibition time of oxygen consumption due to $\alpha$-T alone (10 $\mathrm{min})$ and in combination with ASC (17 $\mathrm{min})$ or 5-ASA (20 $\mathrm{min}$ ), separately or together (26 min). Experimental conditions are described in Section 2. Recordings are representative assays of at least three independent experiments.

tained are shown in Fig. 6, indicating that neither 5-ASA nor ASC have the ability to protect, efficiently, $\alpha$-T from the attack of peroxyl radicals initially generated in the lipid phase. In fact, only a monotonic decrease in the $\alpha$-T consumption rate can be observed when the hydrophilic compounds are added to the aqueous phase. In contrast with the results obtained when peroxyl radicals were formed in the aqueous phase (Fig. 4), no initial inhibition period of the $\alpha$-T consumption is detected. When 5-ASA and ASC are tested together, a higher decrease in the oxidation rate of $\alpha$-T occurs as compared with the rates obtained with each compound individually.

\section{Discussion}

We have previously shown that 5-ASA is highly effective against membrane peroxidation as well as an efficient scavenger of peroxyl radicals (Dinis et al., 1994). Recently, we have also demonstrated that 5-ASA potentiates the antioxidant capacity of vitamin E (Gonçalves et al., 1998). On the other hand, ASC is known to protect $\alpha$-T from oxidation directly or by the $\alpha$-tocopheroxyl radical recycling, an important mechanism of antioxidant capacity of membranes (Bisby and Parker, 1995). Thus, the present study of the combined effects of 5-ASA and ASC on AAPH- or AMVN-induced lipid peroxidation of PC liposomes, with $\alpha$-T previously incorporated, seem interesting to us, since in IBD there is not only an increase in the production of ROS, but also a decrease in the antioxidant defences, particularly in the content of ascorbic acid (Buffinton and Doe, 1995a,b).

5-ASA and ASC are hydrophilic compounds located outside the membranes, in the aqueous

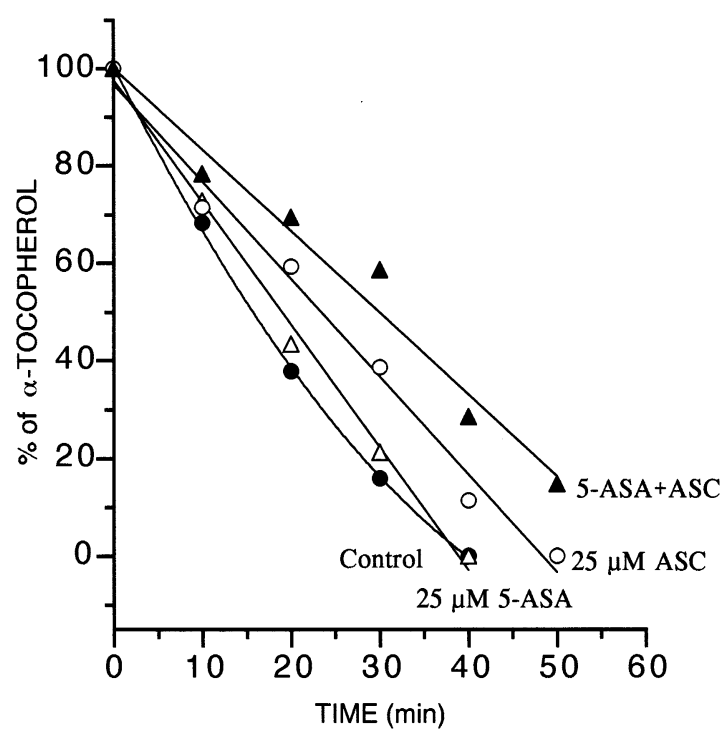

Fig. 6. Rates of $\alpha-\mathrm{T}$ consumption along the oxidation of PC liposomes $(1.5 \mathrm{mM} \mathrm{PC}, 2.5 \mu \mathrm{M} \alpha-\mathrm{T})$ initiated by AMVN (500 $\mu \mathrm{M})$, at $56^{\circ} \mathrm{C}$ in the absence $(\bullet)$ and presence of $25 \mu \mathrm{M}$ 5-ASA $(\triangle), 25 \mu \mathrm{M}$ ASC $(\bigcirc)$ and $25 \mu \mathrm{M}$ 5-ASA $+25 \mu \mathrm{M}$ ASC $(\Delta)$. The points represent the means of three experiments. Deviations are generally encompassed by the size of the symbols. 
region, where the peroxyl radicals are generated from AAPH, at a constant flux (Niki, 1990). Thus, in the concentrations used, 5-ASA and ASC are the first targets of the radical attack, reacting with them independently according to their reactivities. This explains the additive effects observed in the lag phases of oxygen consumption and of production of conjugated dienes when combined, indicating that the antioxidant-derived radical species are devoid of oxidant activity. The measurements of 5-ASA and $\alpha$-T throughout the oxidation reaction confirm the preferential attack of peroxyl radicals to 5-ASA and ASC before starting the $\alpha-\mathrm{T}$ and lipid oxidation, evidencing also a higher reactivity of ASC than of 5-ASA to the radicals, as elicited from the protection afforded by ASC to 5-ASA degradation (Fig. 3).

When the peroxyl radicals are generated initially in the lipophilic domain of the membranes, the results also clearly support an additive interaction between the two hydrophilic compounds in the prolongation of the lag phase of oxygen consumption due to $\alpha$-T (Fig. 5). As has previously been reported, 5-ASA and ASC per se do not protect significantly the membrane lipids from AMVN-induced lipid peroxidation, but potentiate drastically the antioxidant activity of $\alpha-\mathrm{T}$ incorporated in membranes (Niki, 1987; Gonçalves et al., 1998). However, the effectiveness of the 5ASA and ASC combination in extending the inhibition time of the oxidation reaction due to $\alpha$-T is only additive. Neither 5-ASA nor ASC, located in the aqueous phase, are able to efficiently scavenge radicals generated within the lipid region, but decrease the rate of $\alpha$-T consumption (Fig. 6) extending the time required to start lipid oxidation (Fig. 5).

In conclusion, although 5-ASA is chemically less reactive toward peroxyl radicals than ASC, and does not present any synergistic interaction with this endogenous antioxidant at the concentrations used, it may act as a very effective radical scavenging antioxidant, especially in the hydrophilic domain of the membranes. Despite it being debatable whether the enhanced production of ROS, in the colonic mucosa of patients with IBD, is a cause or a consequence of the disease, these species are produced in excess by the infl- amed mucosa, and the efficacy of the treatment will be undoubtedly related with the antioxidant activity of the drug used. Thus, our data clearly point to the beneficial effects of 5-ASA in the treatment of IBD, not only by the direct antioxidant effects related with the scavenging of radical species, but also by the increase in the antioxidant capability of mucosa. Considering the increased oxidative stress and decreased antioxidant defences of mucosa in inflammatory bowel diseases, this dual ability of 5-ASA may be very important for its therapeutic effects.

\section{Acknowledgements}

Supported by PRAXIS XXI Program. E. Gonçalves was a recipient of a grant from PRAXIS XXI (BM/6568/95). The authors thank Professor V.M.C. Madeira for his advice and helpful suggestions.

\section{References}

Ahnfelt-Ronne, I., Nielsen, O.H., Christensen, A., Langholz, E., Binder, V., Riis, P., 1990. Clinical evidence supporting the radical scavenger mechanism of 5-aminosalicylic acid. Gastroenterology 98, 1162-1169.

Allgayer, H., Rang, S., Klotz, U., Bohne, P., Retey, J., Kruis, W., Gugler, R., 1994. Superoxide inhibition following different stimuli of respiratory burst and metabolism of aminosalicylates in neutrophils. Dig. Dis. Sci. 9, 145-151.

Aruoma, O.I., Wasil, M., Halliwell, B., Hoey, B., Butler, J., 1987. The scavenging of oxidants by sulphasalazine and its metabolites. A possible contribution to their anti-inflammatory effects? Biochem. Pharmacol. 36, 3739-3742.

Beyer, R.E., 1994. The role of ascorbate in antioxidant protection of biomembranes: interaction with vitamin $\mathrm{E}$ and coenzyme Q. J. Bioenerg. Biomembr. 26, 349-358.

Bisby, R.H., Parker, A.W., 1995. Reaction of ascorbate with the $\alpha$-tocopheroxyl radical in micellar and bilayer membrane systems. Arch. Biochem. Biophys. 17, 170-178.

Buffinton, G.D., Doe, W.F., 1995a. Altered ascorbic acid status in the mucosa from inflammatory bowel disease patients. Free Radic. Res. 22, 131-143.

Buffinton, G.D., Doe, W.F., 1995b. Depleted mucosal antioxidant defenses in inflammatory bowel disease. Free Radic. Biol. Med. 19, 911-918.

Burton, G.W., Webs, A., Ingold, K.U., 1985. A mild, rapid, and efficient method of lipid extraction for use in determining vitamin E/lipid ratios. Lipids 20, 29-39. 
Conner, E.M., Brand, S.J., Davis, J.M., Kang, D.Y., Grisham, M.B., 1996. Role of reactive metabolites of oxygen and nitrogen in inflammatory bowel disease: toxins, mediators, and modulators of gene expression. Inflamm. Bowel Dis. 2, $133-147$.

Corongiu, F.P., Banni, S., Dessi, M.A., 1989. Conjugated dienes detected in tissue lipid extracts by second derivative spectrophotometry. Free Radic. Biol. Med. 7, 183-186.

Dinis, T.C.P., Madeira, V.M.C., Almeida, L.M., 1994. Action of phenolic derivatives (Acetaminophen, salicylate, and 5-aminosalicylate) as inhibitors of membrane lipid peroxidation and as peroxyl radical scavengers. Arch. Biochem. Biophys. 315, 161-169.

Doba, T., Burton, G.W., Ingold, K.U., 1985. Antioxidant and co-antioxidant activity of vitamin $\mathrm{C}$. The effect of vitamin $\mathrm{C}$, either alone or in the presence of vitamin $\mathrm{E}$ or a water-soluble vitamin $\mathrm{E}$ analogue, upon the peroxidation of aqueous multilamellar phospholipid liposomes. Biochim. Biophys. Acta 835, 298-303.

Fiorentini, D., Cipollone, M., Galli, M.C., Pugnaloni, A., Biagini, G., Landi, L., 1994. Characterization of large unilamellar vesicles as models for studies of lipid peroxidation initiated by azocompounds. Free Radic. Res. 21, 329-339.

Fischer, C., Klotz, U., 1994. Radical-derived oxidation products of 5-aminosalicylic acid and $N$-acetyl-5-aminosalicylic acid. J. Chromatogr. 661, 57-68.

Gonçalves, E., Almeida, L.M., Dinis, T.C.P., 1998. Antioxidant activity of 5-aminosalicylic acid against peroxidation of phosphatidylcholine liposomes in the presence of $\alpha$-tocopherol. A synergistic interaction? Free Radic. Res. (in press).

Grisham, M.B., Granger, D.N., 1988. Neutrophil-mediated mucosal injury. Role of reactive oxygen metabolites. Dig. Dis. Sci. 33, 6S-15S.

Keshavarzian, A., Sedghi, S., Kanofsky, J., List, T., Robinson, C., Ibrahim, C., Winship, D., 1992. Excessive production of reactive oxygen metabolites by inflamed colon: analysis by chemiluminescence probe. Gastroenterology 103, 177185.

Lang, J.K., Gohil, K., Packer, L., 1986. Simultaneous determination of tocopherols, ubiquinols, and ubiquinones in blood, plasma, tissue homogenates, and subcellular fractions. Anal. Biochem. 57, 106-116.

Lih-Brody, L., Powell, S.R., Collier, K.P., Reddy, G.M., Cerchia, R., Kahn, E., Weissman, G., Katz, S., Floyd, R.A., McKinley, M.J., Fisher, S.E., Mullin, G.E., 1996. Increased oxidative stress and decreased antioxidant defenses of inflammatory bowel disease. Dig. Dis. Sci. 41, $2078-2086$.

MacDonald, R.C., MacDonald, R.I., Menco, B., Takeshita, K., Subbarao, N.K., Hu, L., 1991. Small-volume extrusion apparatus for preparation of large, unilamellar vesicles. Biochim. Biophys. Acta 1061, 297-303.

Miles, A.M., Grisham, M.B., 1994. Antioxidant properties of aminosalicylates. Methods Enzymol. 234, 555-572.

Millar, A.D., Rampton, D.S., Chander, C.L., Claxson, A.W.D., Blades, S., Coumbe, A., Panetta, J., Morris, C.J., Blake, D.R., 1996. Evaluating the antioxidant potential of new treatments for inflammatory bowel disease using a rat model of colitis. Gut 39, 407-415.

Nielsen, O.H., Rask-Madsen, J., 1996. Mediators of inflammation in chronic inflammatory bowel disease. Scand. J. Gastroenterol. 31 (Suppl 216), 149-159.

Niki, E., 1987. Antioxidants in relation to lipid peroxidation. Chem. Phys. Lipids 44, 227-253.

Niki, E., 1990. Free radical initiators as source of water- or lipid-soluble peroxyl radicals. Methods Enzymol. 186, 101-108.

Rhodes, J., Thomas, G., Evans, B.K., 1997. Inflammatory bowel disease management. Some thoughts on future drug developments. Drugs 3, 189-194.

Slater, T.F., 1984. Overview of methods used for detecting lipid peroxidation. Methods Enzymol. 105, 283-293.

Tesoriere, L., Bongiorno, A., Pintaudi, A.M., D’Anna, R., D'Arpa, D., Livrea, M.A., 1996. Synergistic interactions between vitamin $\mathrm{A}$ and vitamin $\mathrm{E}$ against lipid peroxidation in phosphatidylcholine liposomes. Arch. Biochem. Biophys. 326, 57-63.

Yamamoto, Y., Niki, E., Kamiya, Y., Shimasaki, H., 1984. Oxidation of lipids. 7. Oxidation of phosphatidylcholine in homogeneous solution and in water dispersion. Biochim. Biophys. Acta 795, 332-340. 